Molecular self-assembly on an insulating surface: interplay between substrate templating and intermolecular interactions

This article has been downloaded from IOPscience. Please scroll down to see the full text article.

2012 J. Phys.: Condens. Matter 24354007

(http://iopscience.iop.org/0953-8984/24/35/354007)

View the table of contents for this issue, or go to the journal homepage for more

Download details:

IP Address: 134.93.38.15

The article was downloaded on 24/08/2012 at 14:53

Please note that terms and conditions apply. 


\title{
Molecular self-assembly on an insulating surface: interplay between substrate templating and intermolecular interactions
}

\author{
Markus Kittelmann, Philipp Rahe ${ }^{1}$ and Angelika Kühnle \\ Institut für Physikalische Chemie, Johannes Gutenberg-Universität Mainz, Duesbergweg 10-14, \\ 55099 Mainz, Germany \\ E-mail: kuehnle@uni-mainz.de
}

Received 27 February 2012, in final form 30 March 2012

Published 16 August 2012

Online at stacks.iop.org/JPhysCM/24/354007

\begin{abstract}
We report on molecular self-assembly of biphenyl-4,4'-dicarboxylic acid (BPDCA) on $\mathrm{CaCO}_{3}(10 \overline{1} 4)$ under ultra-high vacuum conditions. Two-dimensional, ordered islands are obtained upon deposition at room temperature, coexisting with a streaky structure that is ascribed to individual, mobile molecules forming a two-dimensional gas-like phase.

High-resolution non-contact atomic force microscopy (NC-AFM) images of the molecular islands reveal an ordered inner structure that is dominated by rows of molecules aligned side by side running along the [ $\overline{42} 61]$ crystallographic direction. A detailed analysis of these rows exhibits inter-row distances that are multiples of the calcite unit cell dimension along the [01 10$]$ direction, clearly demonstrating the templating effect of the substrate. Our results indicate that an excellent size match of the molecular structure with respect to the underlying substrate results in an increased binding of the BPDCA molecules to the surface. In between the rows, a different molecular structure is coexisting with the molecules aligning head to tail. This structure is explained by intermolecular hydrogen bond formation very similar to the BPDCA bulk structure. The coexistence of the bulk-like structure with the row structure suggests a close balance of intermolecular and molecule-surface interactions to be responsible for the observed structure formation.
\end{abstract}

(Some figures may appear in colour only in the online journal)

\section{Introduction}

Molecular self-assembly on surfaces is governed by a subtle interplay between intermolecular and molecule-surface interactions [1]. In fact, when aiming at exploiting the structural variety of organic molecules for self-assembled structure formation on surfaces, it is important to balance these two interactions, which is frequently achieved for molecular self-assembly on metallic surfaces [2]. On insulating substrates and thin insulating films, however,

1 Present address: Department of Physics and Astronomy, The University of molecule-surface interactions have been found to be considerably reduced as compared to metallic substrates [3-6]. This reduced interaction frequently results in molecular bulk crystal formation [4] or molecular dewetting [7-9] instead of self-assembly of extended two-dimensional islands. Several strategies have been explored to overcome this limitation, including surface patterning $[10,11]$ and increasing the electrostatic interaction for anchoring polar molecules on ionic crystals $[12,5]$. So far, however, only very few examples exist demonstrating the formation of a molecular wetting layer on an insulating surface; tailoring the molecule-surface interaction by optimizing the geometrical match of the molecular dimensions to the crystal lattice has been demon- 
(a)

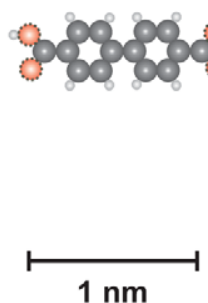

(b)

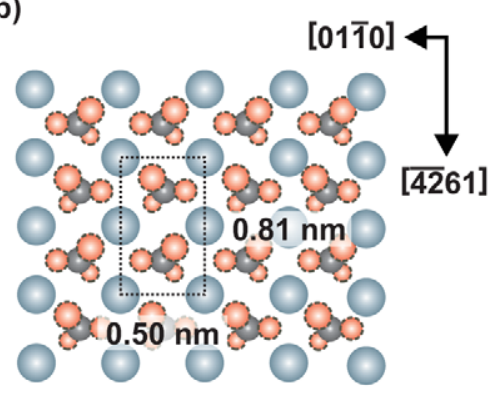

Figure 1. (a) Model of biphenyl-4,4-dicarboxylic acid (BPDCA). (b) Model of the calcite (1014) surface with a rectangular unit cell of $a \times b=0.50 \mathrm{~nm} \times 0.81 \mathrm{~nm}$. The two carbonate groups within the unit cell are rotated with respect to each other.

strated to constitute a versatile strategy for anchoring organic molecules to insulating surfaces and for steering the resulting structure formation [13-16]. For example, a hydrogen-bonded network of benzene diboronic acid molecules has been shown to form an extended wetting layer on $\mathrm{KCl}(001)$ at room temperature [15]. In this study, the optimized molecular structure in the absence of the substrate perfectly matches the crystal lattice registry, nicely explaining the stability of the observed structure. Recently, the high surface energy of calcite has been exploited to form a wetting layer of $\mathrm{C}_{60}$ molecules at room temperature [17].

Here, we present a system that directly expresses the balance between intermolecular and molecule-surface interactions by the coexistence of two different molecular structures on the surface. Using high-resolution non-contact atomic force microscopy (NC-AFM) imaging under ultrahigh vacuum conditions (UHV), we study the molecular self-assembly of biphenyl-4,4'-dicarboxylic acid (BPDCA, figure 1(a)) on the most stable cleavage plane of calcite, $\mathrm{CaCO}_{3}$ (1014) (figure 1(b)) [18], at room temperature. Ordered rows of molecules aligned side by side running along the [4261] crystallographic direction demonstrate a significant molecule-surface interaction that can be explained by an excellent geometrical match of the molecular structure to the substrate dimensions. In between these rows, a second structure is formed with the molecules aligning head to tail, closely resembling the molecular bulk structure. Our results, thus, indicate that molecule-substrate and intermolecular interactions are of similar strength in the presented system.

\section{Methods}

All experiments presented here were carried out in a UHV chamber with a base pressure better than $1 \times 10^{-10}$ mbar. NC-AFM imaging was performed at room temperature using an NC-AFM (VT AFM 25 from Omicron Nanotechnology $\mathrm{GmbH}$, Taunusstein, Germany) operated in the frequency modulation non-contact mode [19]. For signal demodulation and oscillation excitation, a phase-locked loop detector and amplitude controller (easyPLL plus from Nanosurf, Liestal, Switzerland) was used. As force sensors, Ar-sputtered n-doped silicon cantilevers (type PPP NCH from NanoWorld,
Neuchâtel, Switzerland) with resonance frequencies around $300 \mathrm{kHz}$ were used. Typical oscillation amplitudes were in the order of $10 \mathrm{~nm}$. All images shown are carefully corrected for drift by comparing subsequent upwards and downwards images [20].

Calcite crystals of optical quality (Korth Kristalle, Altenholz (Kiel), Germany) were cleaved in situ using a scalpel and home-built sample holder [21]. To remove surface charges present after the cleavage step, crystals were annealed to a maximum temperature of $\sim 480 \mathrm{~K}$ for $1.5 \mathrm{~h}$. The BPDCA molecules were purchased from Aldrich (Munich, Germany, 97\% purity) and used without further purification, except for outgassing at $360 \mathrm{~K}$ for several hours before deposition. The molecules were deposited from a home-built Knudsen cell onto freshly prepared surfaces held at room temperature. Cell temperatures around $410 \mathrm{~K}$ were used, yielding deposition rates in the order of $0.13 \mathrm{ML} \mathrm{min}^{-1}$.

\section{Results and discussion}

A representative NC-AFM topography image obtained after deposition of approximately 0.5 ML BPDCA molecules onto $\mathrm{CaCO}_{3}(10 \overline{1} 4)$ held at room temperature is given in figure 2(a). In this image, molecular islands are observed that are separated by streaky regions. The islands exhibit an elongated shape with typical dimensions of a few tens of nanometer in the [0110] direction and of $10-30 \mathrm{~nm}$ in the [4261] direction. The apparent height of the islands is approximately $0.25 \mathrm{~nm}$ (measured in images with smaller scan size-not shown), suggesting that the islands are formed by flat-lying molecules. The edges of the islands appear fuzzy, which is a typical sign for attachment and detachment of molecules at the islands' periphery. The streaky regions and the existence of the islands indicate that individual molecules (i) can detach from the islands and (ii) have substantial mobility on the surface at room temperature to form a two-dimensional gas-like phase.

An internal structure is revealed on the islands, which is predominantly composed of rows running along the [4261] direction. The inner structure is resolved in the frequency shift image shown in figure 2(b). Most prominently, the ordered rows running along the [4261] direction are observed to be composed by features that fit in size to flat-lying, individual molecules aligned side by side. 

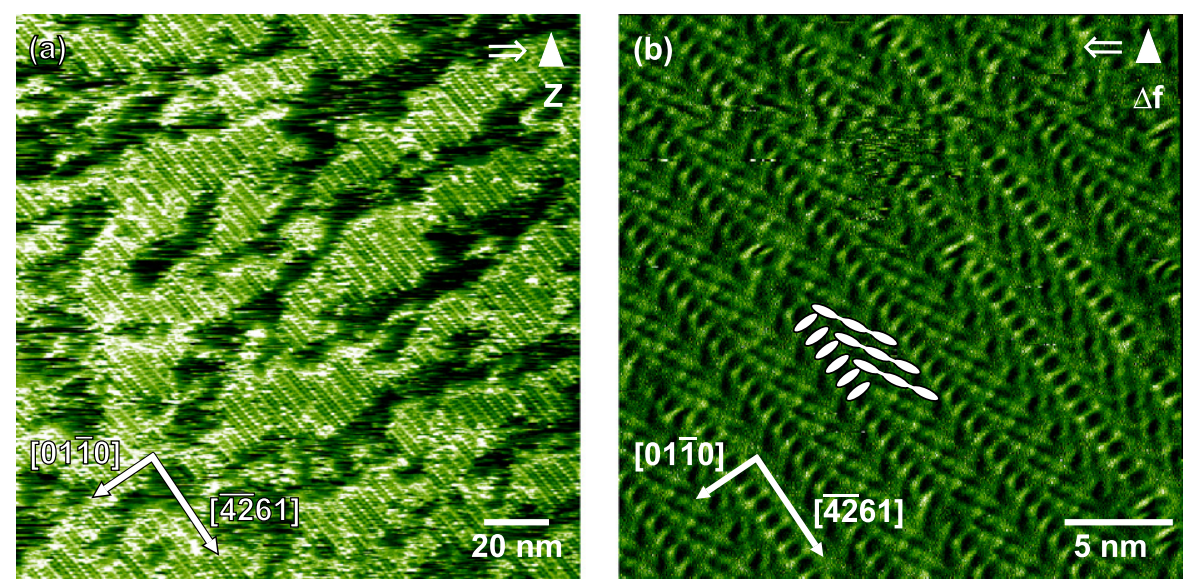

Figure 2. NC-AFM images showing BPDCA islands on $\mathrm{CaCO}_{3}(10 \overline{1} 4)$ held at room temperature. (a) Topography image exhibiting BPDCA islands separated by streaky regions. (b) Frequency shift image taken on a molecular island, revealing the internal structure. Molecules aligning in side-by-side rows and head-to-tail rows are indicated by ellipses.

Interestingly, the separation of these side-by-side rows along the [01 10 ] direction is not equidistant, but shows different distances. The area in between the side-by-side rows is occupied by another structure with the molecules aligning head to tail, forming a characteristic angle of $27^{\circ}$ with respect to the side-by-side row structure.

To analyze the molecular islands in more detail, a high-resolution frequency shift image is given in figure 3(a) along with a model for the molecular arrangement shown in figure 3(b). Three different inter-row distances are marked within this image, exhibiting distances of 3.0, 3.5 and $4.0 \mathrm{~nm}$, respectively. Interestingly, these distances appear as multiples of the unit cell dimension of $0.5 \mathrm{~nm}$ along the [01 10 ] direction, indicating that the surface structure has a decisive influence on the formation of these side-by-side rows. In order to quantify this observation, we have analyzed a total number of 224 inter-row distances from four independent large-scale images. The resulting distance histogram is shown in figure 3(c). As can be seen, a discrete distribution is, indeed, obtained exhibiting inter-row spacings that are multiples of the unit cell dimension of $0.5 \mathrm{~nm}$. Moreover, a clear maximum at an inter-row spacing of $3.5 \mathrm{~nm}$ is revealed, which will be explained later.

From our images, we can deduce the orientation of the molecules within the side-by-side rows with respect to the underlying calcite lattice. The absolute adsorption position is, however, not obtained experimentally as simultaneous imaging of the molecular islands and the calcite lattice was hampered by the molecular mobility at room temperature. Based on simple electrostatic considerations, we can identify two very likely adsorption positions that might be present in the experimental data shown in figure 3(a). We stress, however, that more elaborate calculations are required for confirming the qualitative discussion made here. Nevertheless, our conclusions regarding the molecular arrangement and the most likely inter-row spacing remain valid regardless of the precise adsorption. Two obvious adsorption positions of the molecules within the side-by-side rows are shown in figure 3(d). The models are based on the fact that the molecule-surface interaction is steered by the interaction of the carboxylic groups with the surface [22]. The hydrogen atom of the carboxylic groups anchors to a surface oxygen atom via a hydrogen bond, while the partially negatively charged carbonyl oxygen atom is positioned close to a surface calcium cation. When aligning the molecular axis along the [01 $\overline{1} 0]$ direction as observed experimentally, the size of the molecule allows for the accommodation of both carboxylic groups on top of equivalent adsorption positions. This explains the strong surface templating effect, as both carboxylic groups can attain an energetically favorable adsorption position. Depending on the position of the hydrogen atom, two slightly differing adsorption positions can be obtained. In the upper part of figure 3(d), two molecules are shown (labeled 1 and 2) with both hydrogen atoms arranged in the cis configuration. In this case, the molecule can be accommodated such that main axis of the molecule is exactly perpendicular to the side-by-side row direction. However, a second option would be to rotate one carboxylic acid around the $\mathrm{C}-\mathrm{C}$ axis with the hydrogen atoms adopting the trans configuration (shown in the molecules labeled 3 and 4). In this case, the molecular axis would form an angle of approximately $5^{\circ}$ with respect to the side-by-side row. This slightly rotated configuration might be evident for the rows shown in figure 3(a) except for the one marked with an A.

The ordering within the rows is very well-pronounced, and defects can be found only rarely. One defect is shown in figure 3(a) (marked by a circle), which originates from a shift of one side-by-side row by $0.5 \mathrm{~nm}$. The linking molecule appears to bridge the two rows in a tilted fashion.

The area in between the rows is occupied by molecules forming a distinctly different pattern. In this structure, the molecules are found to align head to tail, such that the carboxylic groups face each other. This arrangement allows for hydrogen bond formation between the carboxylic groups and is very close to what is expected in the BPDCA bulk structure [23]. The observed ordering is a clear sign for the fact that the molecules are not deprotonated yet, which is in agreement with the $\mathrm{p} K_{\mathrm{a}}$ values of 3.5 and 4.3 , respectively [24]. 

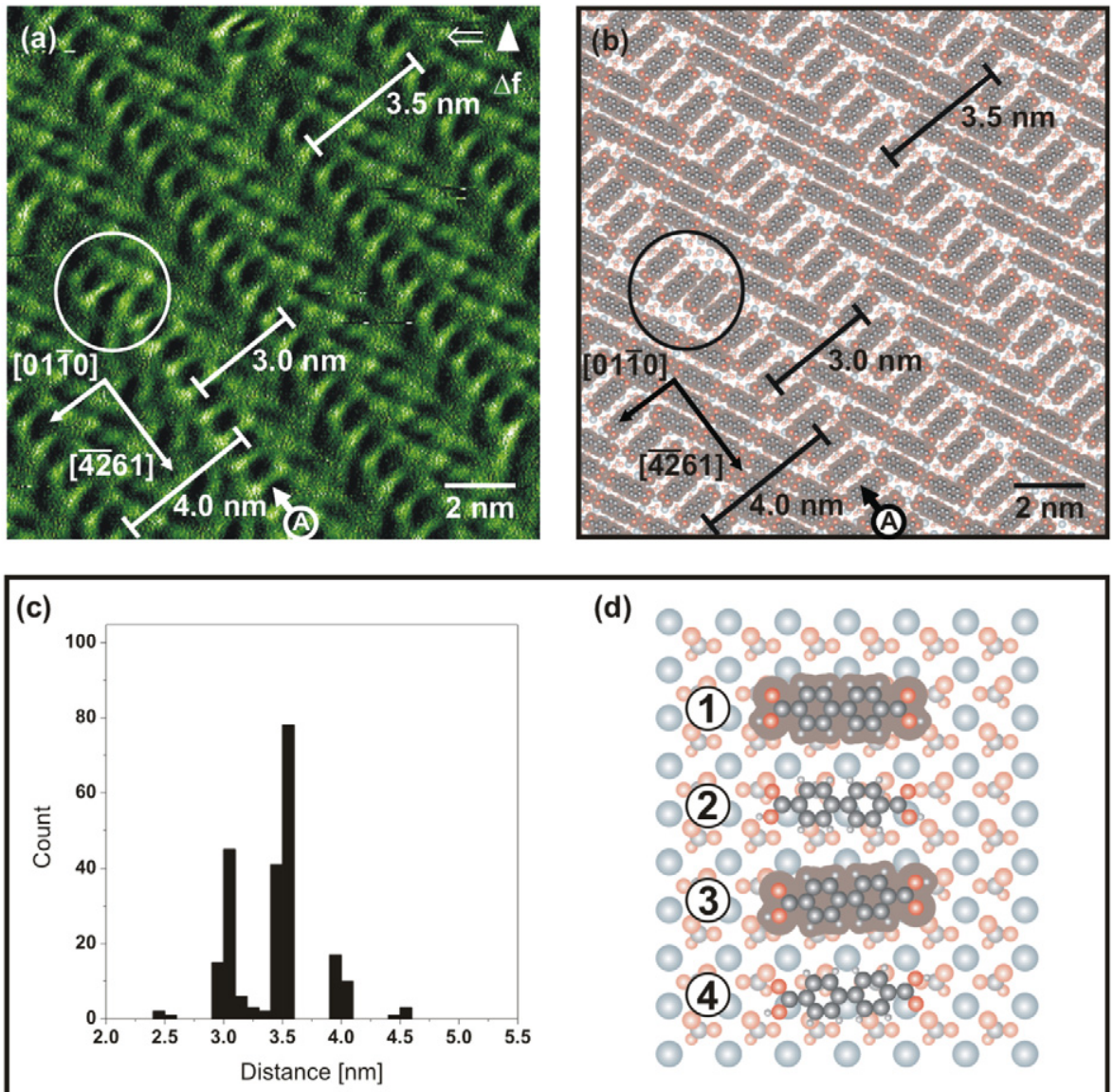

(d)

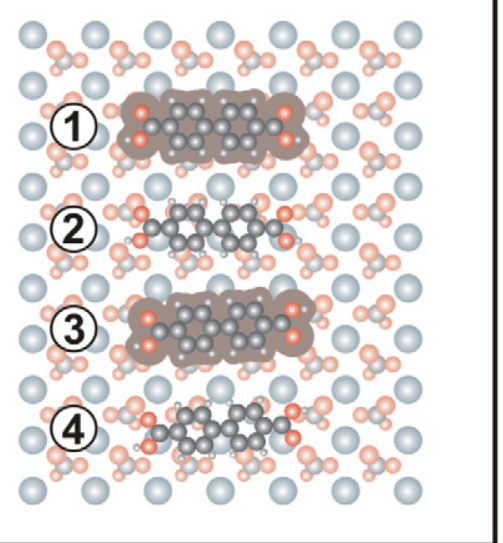

Figure 3. Detailed analysis of a BPDCA island. (a) NC-AFM frequency shift image. A defect (circle) and three different inter-row distances are marked. (b) Model visualizing the molecular arrangement as present in (a). The gray region around the molecules corresponds to the van der Waals radius. (c) Histogram showing the distribution of 224 inter-row distances from four independent images. (d) Possible adsorption positions of the molecules within the side-by-side rows with respect to the underlying substrate.

The lengths of these head-to-tail rows differ depending on the available space between the above-mentioned side-by-side rows running along the $[\overline{42} 61]$ direction. For inter-row distances of $3.0,3.5$ and $4.0 \mathrm{~nm}$, an alignment of two, three and four molecules is revealed, respectively, as shown in figure 4(a) and listed in table 1. The 'head' and 'tail' molecules from a head-to-tail row arrange in a way that they can form hydrogen bonds towards the oxygen atom of the neighboring molecule within the adjacent side-by-side row, while the partially negatively charged oxygen atom is close to a surface calcium cation. This configuration explains the experimentally observed characteristic angle of $27^{\circ}$.

To analyze the obtained inter-row spacing histogram from figure 3(c) in more detail, we calculated the length of a head-to-tail row and compared this length with the available space between the side-by-side rows. To estimate the length of a head-to-tail row, we added the estimated molecule length $(\mathrm{O}-\mathrm{O}$ distance: $1.15 \mathrm{~nm}$ [23]) and hydrogen bonds length (O-O distance: $0.27 \mathrm{~nm} \mathrm{[25])} \mathrm{as} \mathrm{listed} \mathrm{in} \mathrm{the}$ third column in table 1 . We want to stress that (i) these numbers do not include the interaction with the surface and (ii) all hydrogen bonds were considered to be of the same length, regardless of their nature (within the head-to-tail row or between the head-to-tail row and the side-by-side row).
Table 1. Comparison of head-to-tail row lengths with available space for the respective side-by-side inter-row distances.

\begin{tabular}{llll}
\hline $\begin{array}{l}\text { Side-by-side } \\
\text { inter-row } \\
\text { distance }(\mathrm{nm})\end{array}$ & $\begin{array}{l}\text { Number of } \\
\text { molecules in } \\
\text { head-to-tail } \\
\text { row }\end{array}$ & $\begin{array}{l}\text { Head-to-tail } \\
\text { row length } \\
\text { calculated } \\
(\mathrm{nm})\end{array}$ & $\begin{array}{l}\text { Space } \\
\text { available } \\
\text { (distance) } \\
(\mathrm{nm})\end{array}$ \\
\hline 2.5 & 1 & 1.69 & 1.94 \\
3.0 & 2 & 3.11 & 3.40 \\
3.5 & 3 & 4.53 & 4.68 \\
4.0 & 4 & 5.95 & 5.94 \\
4.5 & 5 & 7.37 & 7.21 \\
\hline
\end{tabular}

Thus, the given length values represent a rough estimate only. We compare these lengths with the space available between the side-by-side rows (considering the distance between two oxygen atoms as indicated in figure 4(a)) listed in the fourth column. As can be seen, for head-to-tail rows consisting of only one or two molecules, the available space is considerably larger than the estimated molecule length, indicating that the interaction of the head-to-tail rows with the side-by-side rows is weak or even absent. For head-to-tail rows consisting of five molecules, the distance between the side-by-side rows is smaller than what would be needed to easily accommodate 


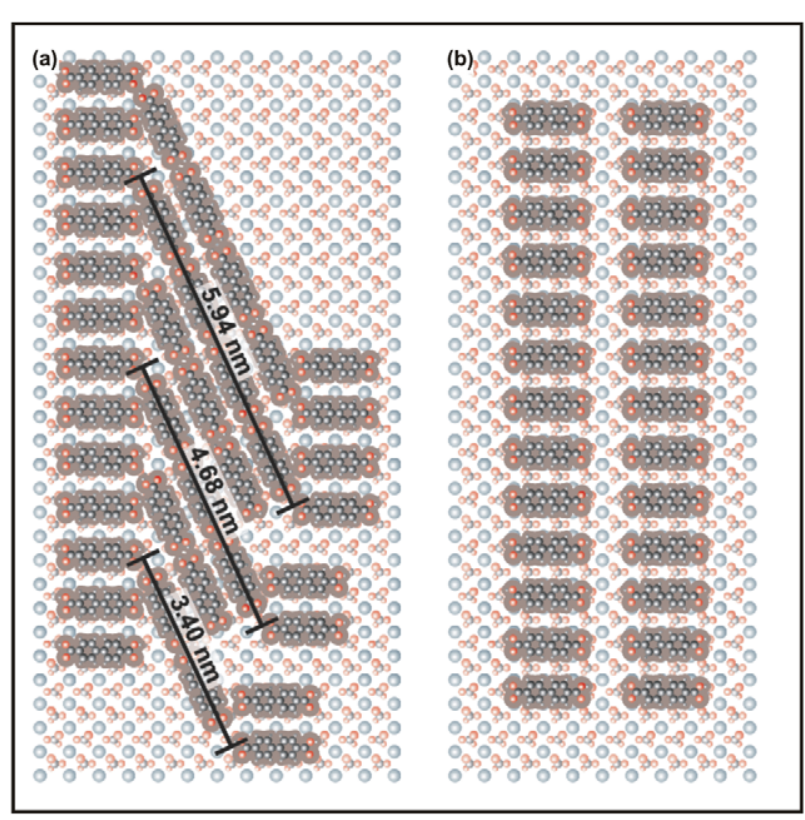

Figure 4. Models illustrating possible molecular arrangements. (a) Model showing both the side-by-side rows and the head-to-tail rows. Different lengths of head-to-tail rows are indicated, composed of two, three and four molecules, respectively. (b) Closest alignment of a pure side-by-side row phase, revealing a large spacing between the molecular rows.

the head-to-tail rows. For head-to-tail rows consisting of three and four molecules, the available space is very close to the estimated space required, suggesting that these head-to-tail rows should be favored. Indeed, our experimental results reveal a maximum at an inter-row distance of $3.5 \mathrm{~nm}$ in figure 3(c). We, therefore, explain the maximum in the histogram in figure 3(c) by a favorable size match in the inter-row distance and the space required for the head-to-tail rows.

Finally, we comment on the situation that would arise in the absence of the head-to-tail rows. Because of the well-defined adsorption position of the molecules within the side-by-side rows, the most close-packed arrangement is associated with a rather large spacing between the molecular rows, as shown in figure 4(b). Thus, no inter-row interaction is expected in this configuration, which is unfavorable compared to the situation where the head-to-tail rows interlink the side-by-side rows.

\section{Summary and conclusions}

The coexistence of two different molecular adsorption configurations is shown upon deposition of BPDCA on $\mathrm{CaCO}_{3}(10 \overline{1} 4)$. A very pronounced row structure is revealed that is formed by flat-lying molecules aligned side by side, resulting in rows running along the [ $\overline{42} 61]$ direction. In this configuration, the molecules show a favorable adsorption position with both carboxylic groups anchoring towards the calcite surface. This strong surface templating effect is possible because of the excellent size match of the molecule to the substrate lattice. Besides this structure, another structure is coexisting, formed by rows that are composed of molecules aligned head to tail. In this configuration, the molecules can form hydrogen bonds as present in the bulk structure. The coexistence of these two different adsorption structures indicates a subtle balance between substrate templating on the one hand and molecular bulk crystal formation on the other.

\section{Acknowledgments}

This work was supported by the German Research Foundation (DFG) through the SFB 625 grant TP B17. We gratefully acknowledge most stimulating discussions with André Gourdon.

\section{References}

[1] Barth J V 2007 Annu. Rev. Phys. Chem. 58375

[2] Kühnle A 2009 Curr. Opin. Colloid Interface Sci. 14 157-68

[3] Nony L, Bennewitz R, Pfeiffer O, Gnecco E, Baratoff A, Meyer E, Eguchi T, Gourdon A and Joachim C 2004 Nanotechnology 15 S91

[4] Kunstmann T, Schlarb A, Fendrich M, Wagner T, Möller R and Hoffmann R 2005 Phys. Rev. B 71121403

[5] Such B, Trevethan T, Glatzel T, Kawai S, Zimmerli L, Meyer E, Shluger A L, Amijs C H M, de Mendoza P and Echavarren A M 2010 ACS Nano 4 3429-39

[6] Bombis C, Kalashnyk N, Xu W, Lægsgaard E, Besenbacher F and Linderoth T R 2009 Small 5 2177-82

[7] Burke S A, Mativetsky J M, Hoffmann R and Grütter P 2005 Phys. Rev. Lett. 94096102

[8] Burke S A, Topple J M and Grütter P 2009 J. Phys.: Condens. Matter 21423101

[9] Körner M, Loske F, Einax M, Kühnle A, Reichling M and Maass P 2011 Phys. Rev. Lett. 107016101

[10] Nony L, Gnecco E, Baratoff A, Alkauskas A, Bennewitz R, Pfeiffer O, Maier S, Wetzel A, Meyer E and Gerber C 2004 Nano Lett. 4 2185-9

[11] Mativetsky J M, Burke S A, Fostner S and Grütter P 2007 Small 3 818-21

[12] Schütte J, Bechstein R, Rohlfing M, Reichling M and Kühnle A 2009 Phys. Rev. B 80205421

[13] Dienel T, Loppacher C, Mannsfeld S C B, Forker R and Fritz T 2008 Adv. Mater. 20959

[14] Burke S A, Ji W, Mativetsky J M, Topple J M, Fostner S, Gao H J, Guo H and Grütter P 2008 Phys. Rev. Lett. 100186104

[15] Pawlak R, Nony L, Bocquet F, Olson V, Sassi M, Debierre J M, Loppacher C and Porte L 2010 J. Phys. Chem. C 114 9290-5

[16] Glatzel T, Zimmerli L, Kawai S, Meyer E, Fendt L A and Diederich F 2011 Beilstein J. Nanotechnol. 2 34-9

[17] Rahe P, Lindner R, Kittelmann M, Nimmrich M and Kühnle A 2012 Phys. Chem. Chem. Phys. 14 6544-8

[18] Rahe P, Schütte J and Kühnle A 2012 J. Phys.: Condens. Matter 24084006

[19] Albrecht T R, Grütter P, Horne D and Rugar D 1991 J. Appl. Phys. $69668-73$

[20] Rahe P, Bechstein R and Kühnle A 2010 J. Vac. Sci. Technol. B 28 C4E 31

[21] Tröger L, Schütte J, Ostendorf F, Kühnle A and Reichling M 2009 Rev. Sci. Instrum. 80063703

[22] Duffy D M and Harding J H 2002 J. Mater. Chem. 12 3419-25

[23] Zhu N, Osada T and Komeda T 2007 Surf. Sci. 601 1789-94

[24] Kittelmann M, Rahe P, Nimmrich M, Hauke C M, Gourdon A and Kühnle A 2011 ACS Nano 5 8420-5

[25] Sledz M, Janczak J and Kubiak R 2001 J. Mol. Struct. $\mathbf{5 9 5} 77-82$ 